\title{
Impact of the amount and type of fat and carbohydrate on vascular function in the RISCK study
}

\author{
Tom Sanders ${ }^{1}$, Fiona Lewis ${ }^{1}$, Gary Frost ${ }^{2}$, Louise Goff ${ }^{2}$ and Phil Chowienczyk ${ }^{1}$ \\ ${ }^{1}$ King's College London, London, UK and ${ }^{2}$ Imperial College London, London, UK
}

RISCK is a multi-centre randomised controlled dietary intervention in subjects at increased risk of metabolic syndrome ${ }^{(1)}$. The results are reported of measurements of vascular function from a substudy conducted on cohorts recruited at King's College London and Imperial College London. Altered vascular function is one of the hallmarks of metabolic syndrome ${ }^{(2)}$. It is currently believed that the impaired vascular function is a consequence of increased production of reactive oxygen species that decrease the bioavailability of NO, a potent anti-atherogenic molecule with vasodilator, anti-platelet, anti-leucocyte and anti-proliferative actions ${ }^{(3)}$. Following a 1-month run-in period on a diet high in SFA, measurements were made of: endothelial function using the flow-mediated dilatation (FMD) technique; endothelial-independent vasodilation following $25 \mu \mathrm{g}$ sublingual glycerol trinitrate (GTN); aortic stiffness using the carotid-femoral pulse wave velocity (PWV); vascular tone using the digital volume pulse stiffness index (SI). Further measures were carried out following a 6-month dietary intervention with a SFA-rich diet similar to the run-in diet, a high-MUFA diet or a low-fat (LF) diet. The target intake for total fat was $38 \%$ energy ( $\%$ E) for the SFA and MUFA diets and $28 \% \mathrm{E}$ for the LF diets. The MUFA and LF diets were designed to reduce dietary SFA to $10 \% \mathrm{E}$ with a planned MUFA intake of $18 \% \mathrm{E}$ on the MUFA diet. The study had statistical power to detect a change of $1 \%$ in FMD. The results are shown in the Table.

\begin{tabular}{|c|c|c|c|c|c|c|}
\hline & \multicolumn{2}{|c|}{ SFA } & \multicolumn{2}{|c|}{ MUFA } & \multicolumn{2}{|c|}{ LF } \\
\hline & Mean & SD or $95 \% \mathrm{CI}$ & Mean & SD or $95 \% \mathrm{CI}$ & Mean & SD or $95 \% \mathrm{CI}$ \\
\hline FMD (\%) & (n 29) & & $(n 44)$ & & (n 37) & \\
\hline Baseline & 6.6 & 2.2 & 6.9 & 2.3 & 6.6 & 2.2 \\
\hline Follow-up & 6.9 & 2.3 & 6.7 & 2.4 & 6.7 & 2.4 \\
\hline Change & 0.3 & $-0.5,1.1$ & -0.2 & $-1.0,0.5$ & 0.1 & $-0.4,0.7$ \\
\hline GTN (\%) & (n 29) & & $(n 44)$ & & (n 36) & \\
\hline Baseline & 11.3 & 3.2 & 11.4 & 3.1 & 11.6 & 4.4 \\
\hline Follow-up & 11.1 & 3.6 & 11.7 & 3.2 & 11.3 & 3.8 \\
\hline Change & -0.2 & $-1.4,0.9$ & 0.3 & $-0.8,1.4$ & -0.3 & $-1.7,1.1$ \\
\hline PWV (m/s) & ( $n$ 27) & & $(n 42)$ & & ( $n$ 36) & \\
\hline Baseline & 8.2 & 1.6 & 7.7 & 1.8 & 7.6 & 1.3 \\
\hline Follow-up & 8.0 & 1.5 & 7.8 & 1.5 & 7.5 & 1.3 \\
\hline Change & -0.2 & $-0.6,0.2$ & 0.2 & $-0.2,0.5$ & -0.1 & $-0.4,0.3$ \\
\hline $\mathrm{SI}(\mathrm{m} / \mathrm{s})$ & ( $n$ 27) & & $(n 41)$ & & ( $n$ 35) & \\
\hline Baseline & 7.9 & 1.6 & 8.1 & 2.0 & 8.1 & 2.3 \\
\hline Follow-up & 8.5 & 2.0 & 8.1 & 2.1 & 7.5 & 1.9 \\
\hline Change & 0.6 & $-0.2,1.3$ & -0.1 & $-0.7,0.5$ & -0.6 & $*-1.2,0.1$ \\
\hline
\end{tabular}

Value was significantly different from that for the SFA treatment (Bonferroni's multiple comparison test): $* P<0.05$.

The dietary intervention did not affect endothelium-dependent or -independent vasodilatation of the brachial artery as assessed by ultrasound. This finding suggests that the diets did not affect the production of NO. Large-artery stiffness as measured by PWV was not affected by treatment. However, there was a borderline-significant $(P=0.05)$ difference between the SFA diet and the LF diet on SI, which although it is correlated with PWV is also an indicator of small-vessel reactivity. The findings of the present study do not support previous conclusions that a diet high in SFA impairs FMD by about $50 \%$ compared with high-MUFA or LF diets in healthy subjects.

This project was funded by the BBSRC and by the Food Standards Agency.

1. Jebb SA, Frost G, Griffin BA, Lovegrove J, Moore C, Sanders T \& Williams C (2007) Nutr Bull 32, 154-156.

2. Williams IL, Wheatcroft SB, Shah AM \& Kearney MT (2002) Obesity, atherosclerosis and the vascular endothelium: mechanisms of reduced nitric oxide bioavailability in obese humans. Int J Obes Relat Metab Disord 26(6) 754-764. 